\title{
An improved pulsed electroacoustic system for space charge measurements under high temperatures and arbitrary waveform voltages
}

Cite as: Rev. Sci. Instrum. 90, 105112 (2019); https://doi.org/10.1063/1.5113693

Submitted: 06 June 2019. Accepted: 04 October 2019 . Published Online: 28 October 2019

Siyu Zhang (D), George Chen (D), Zhiqiang Xu (D), Jiaqi Yan (D), Zongren Peng (D), and Peng Liu (D)

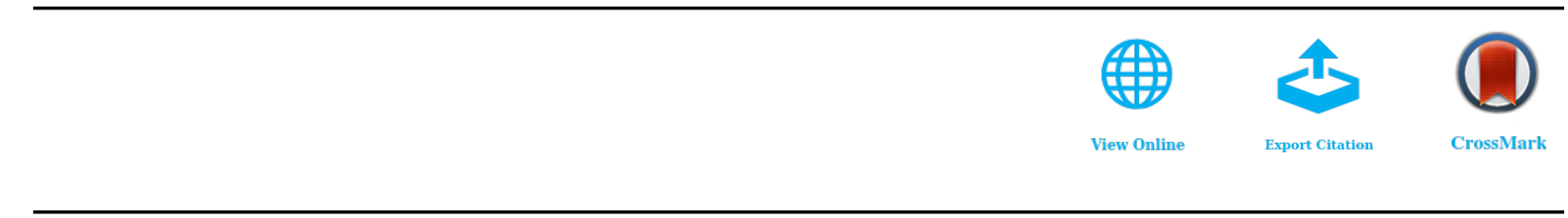

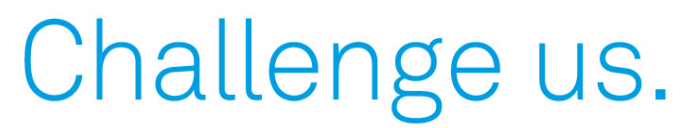

What are your needs for periodic signal detection?

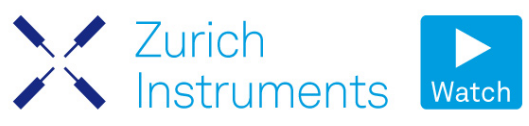

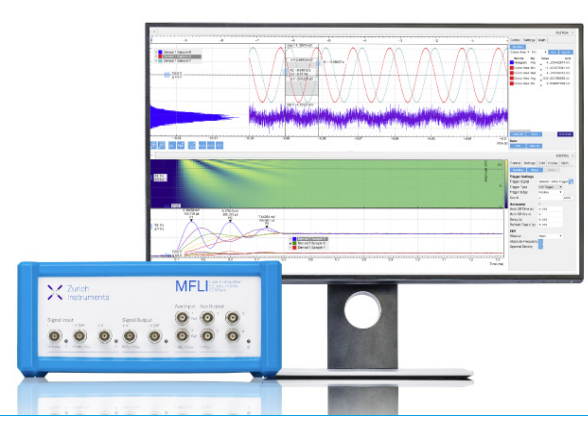

90, 105112 


\title{
An improved pulsed electroacoustic system for space charge measurements under high temperatures and arbitrary waveform voltages
}

\author{
Cite as: Rev. Sci. Instrum. 90, 105112 (2019); doi: 10.1063/1.5113693 \\ Submitted: 6 June 2019 Accepted: 4 October 2019 • \\ Published Online: 28 October 2019
}

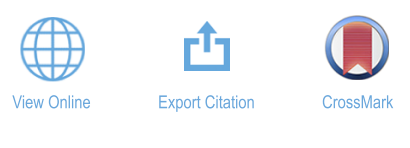

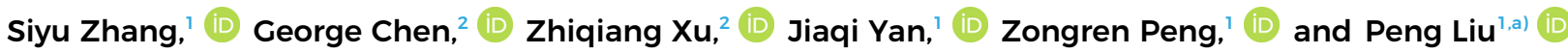

AFFILIATIONS

${ }^{1}$ State Key Laboratory of Electrical Insulation and Power Equipment, Xi'an Jiaotong University, Xi'an 710049, China

${ }^{2}$ School of Electronics and Computer Science, University of Southampton, Southampton SO17 1BJ, United Kingdom
\end{abstract}

${ }^{a)}$ Author to whom correspondence should be addressed: liupeng-cn@mail.xjtu.edu.cn

\begin{abstract}
This paper presents the design and calibration method of an improved pulsed electroacoustic (PEA) system for space charge measurements, including a novel nanosecond pulse generator and an improved electrode system, which can be used under various temperatures and arbitrary waveform voltages. The measurement principle of the PEA system is first analyzed, and the calibration method for the applied voltage is proposed to solve the problem of waveform distortion caused by voltages with different frequency components. For the pulse generator, the main parameters of output pulses are an amplitude of $\sim 700 \mathrm{~V}$, a full width of $4.6 \mathrm{~ns}$, a jitter of $0.24 \mathrm{~ns}$, and a repetition rate of $10 \mathrm{kHz}$ on the $50 \Omega$ load. For the electrode system, the flexible upper electrode design is first introduced to achieve consistently close contact between the sample and the electrode. Besides, the hybrid heating method and the replaceable coupling capacitor and high voltage electrode head are adopted. Space charge measurements are conducted at different conditions using this PEA system, which includes different temperatures and different voltage waveforms. The experimental results verify the effectiveness of the calibration method and the feasibility of the improved PEA system.
\end{abstract}

Published under license by AIP Publishing. https://doi.org/10.1063/1.5113693

\section{INTRODUCTION}

High voltage direct current (HVDC) systems have many irreplaceable advantages, such as higher stability, lower loss, and more economy compared to high voltage alternating current (HVAC) systems. $^{1-3}$ Many pieces of equipment in the HVDC system operate at very complicated conditions, which includes high temperatures, AC-DC combined voltages, and even high frequency harmonic waves. ${ }^{4-6}$ Understanding the space charge characteristics of the insulation materials under these special circumstances is critical to the prevention of field distortion and potential damage to the electrical equipment. ${ }^{7-9}$ Therefore, the precise measurement of charge dynamics inside insulation materials at different temperatures with complex waveform voltages applied is very necessary.

The pulsed electroacoustic (PEA) method was first proposed by Takada in 1983 and has become widely used for space charge measurements in insulation materials. The basic principle is as follows: with the excitation of an impulse voltage, the charges inside the sample are stimulated and an acoustic wave is generated, which can be detected and converted into electrical signals by a piezoelectric sensor. By analyzing the electrical signals, the distribution of space charges inside the sample can be obtained. ${ }^{10,11}$ Measurements were usually carried out on plate samples or cables at room temperature and under DC voltages. ${ }^{12-14}$ Efforts have been made to develop PEA systems that are suitable for different occasions and a significant improvement has been made. On the one hand, to enlarge the temperature scope, band heaters were placed around the test cavity or the test cavity was placed in an oven for heating. ${ }^{15,16}$ Then, space charge measurements under a temperature gradient were realized by adopting a double-circulating-bath design. ${ }^{17-19}$ Even though a stable temperature environment can be achieved, the efficiency of heating could be further increased. On the other hand, for measurements under AC voltages, two factors need to be taken into consideration, which are the measurement of charge profiles at different phase angles and the phase synchronization between the stimulating pulses and the applied voltage, respectively. 
At first, pulses were only applied to the sample at a certain phase angle in each test due to the relatively low repetition rate of the pulse generator. ${ }^{20}$ Then, the pulse repetition rate was increased to $3 \mathrm{kHz}$ and the eclipse system was adopted to increase the data acquisition speed. ${ }^{21}$ The automatic equal phase shift principle was also proposed to obtain charge profiles at more phase angles through measurements in more cycles. ${ }^{22}$ To obtain a higher phase resolution in one cycle, pulse generators with a higher repetition rate should be developed. ${ }^{23-27}$ Besides, similar phenomena, such as the unsynchronized influence charge density and the applied voltage, were observed in many AC space charge experiments while the reasons were not clear. In these tests, the deviation of phase angle ranged from $15^{\circ}$ to $50^{\circ}$, which increased with the increasing frequency. Apart from the explanations in the papers, like the influence of injected charges, it is important to note that the equivalent electric circuit of the measurement system under AC voltages is completely different from that under DC voltages, which might result in a great difference between the applied voltage and the voltage undertaken by the sample. This problem is worse when the applied voltage contains complex frequency components. In addition to the problems of heating and AC testing, special attention should be paid to the consistency of contact between the electrodes and the sample, which influences the transmission of acoustic signals but was rarely discussed before.

In order to measure space charge distributions under high temperatures and complex voltages, an improved heating efficiency, a higher sampling rate, an accurate method of voltage calibration, and a good consistency of contact should be achieved. This paper first presents the theoretical analysis on how different kinds of voltage waveforms influence the voltage undertaken by the sample, based on which the calibration method for the application of arbitrary waveform voltages is proposed. Second, the design principle and test results of the novel pulse generator are introduced in detail. Third, the modified structure of the electrode system is explained, which includes a flexible upper electrode, an oil circulation bath, and the electric heating wires installed in the lower electrode. The new PEA electrode system can greatly improve the efficiency of heating and the consistency of measurements.

\section{THEORETICAL ANALYSIS}

The typical PEA measurement system consists of four parts, the nanosecond pulse generator, the high voltage source, the electrode system, and the piezoelectric sensor. The principle of the PEA method can be briefly summarized as follows: A plate sample is placed between two electrodes. The applied nanosecond pulses generate electric stresses that stimulate small vibrations of space charges and form acoustic pressure waves in each charge layer. The acoustic signals are transmitted through the sample and the electrode to the piezoelectric sensor, which are later transformed into voltage signals. After data calibration and recovery, the charge distribution characteristics inside the sample can be obtained. $^{10,1}$

The equivalent electric circuit for the measurement system is shown in Fig. 1. High voltage $V_{\mathrm{s}}$ and pulse voltage $V_{\mathrm{p}}$ are applied to the sample simultaneously. The sample can be regarded as a

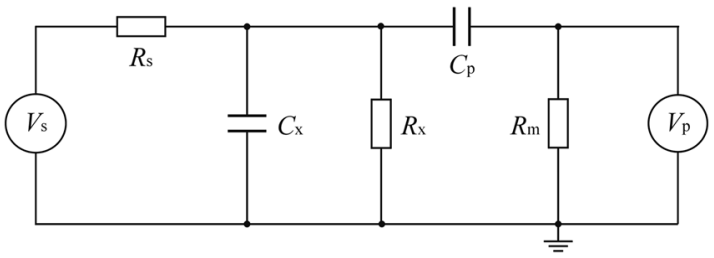

FIG. 1. The equivalent electric circuit of the PEA measurement system.

capacitor $C_{\mathrm{x}}$ in parallel to a resistor $R_{\mathrm{x}}$. The current limiting resistor $R_{\mathrm{S}}$ is used for protection of the high voltage source from overcurrents in case the sample breakdowns. The blocking capacitor $C_{\mathrm{p}}$ is used for protection of the pulse source from the high voltage source. A $50 \Omega$ matched resistor $R_{\mathrm{m}}$ is used to prevent the reflection of pulse voltages at the end of the cable. From Fig. 1, the values of capacitance $C_{\mathrm{p}}$ and resistance $R_{\mathrm{s}}$ will influence the division characteristics of the high voltage and the pulse voltage. Thus, $C_{\mathrm{p}}$ and $R_{\mathrm{s}}$ should be chosen after careful calculation in order to achieve high efficiency, which means that most proportion of the voltages is undertaken by the sample. Even if $C_{\mathrm{p}}$ and $R_{\mathrm{s}}$ are carefully chosen, there still might be some differences between the voltage undertaken by the sample and the applied high voltage $V_{\mathrm{s}}$ when a high frequency AC component is included, which may result in a lowered amplitude and a phase shift from the expected voltage. Therefore, a voltage calibration method is needed to adjust the applied voltage. These two issues are addressed one by one as follows.

Based on the superimposition theorem, the voltage division characteristics of high voltage $V_{\mathrm{s}}$ and pulse voltage $V_{\mathrm{p}}$ are considered separately. When the pulse voltage is solely applied, the equivalent circuit is as shown in Fig. 2(a). Since the resistance of the sample $R_{\mathrm{X}}$, usually in the order of $10^{16} \Omega$, is much larger than the resistance $R_{\mathrm{s}}$, usually in the order between $10^{6}$ and $10^{8} \Omega$, the branch of $R_{\mathrm{X}}$ is neglected. For pulses with a rising time smaller than $5 \mathrm{~ns}$, its equivalent frequency $f_{\mathrm{p}}$ is larger than $100 \mathrm{MHz}$. Since the capacitance $C_{\mathrm{x}}$ is in the order of $10^{-11}$ to $10^{-12} \mathrm{~F}$, its impedance is in the order of $10^{-3}$ to $10^{-4} \Omega$, which makes the branch of $R_{\mathrm{s}}$ also neglectable. The pulse voltage undertaken by the sample is

$$
\overrightarrow{U_{\mathrm{x}}}=\overrightarrow{U_{\mathrm{P}}} \times \frac{1 /\left(j 2 \pi f_{\mathrm{p}} C_{\mathrm{x}}\right)}{1 /\left(j 2 \pi f_{\mathrm{p}} C_{\mathrm{p}}\right)+1 /\left(j 2 \pi f_{\mathrm{p}} C_{\mathrm{x}}\right)}=\overrightarrow{U_{\mathrm{P}}} \times \frac{C_{\mathrm{p}}}{C_{\mathrm{x}}+C_{\mathrm{p}}} \geq 0.8 \overrightarrow{U_{\mathrm{P}}}
$$

For more than $80 \%$ of the pulse voltage to be undertaken by the sample $C_{\mathrm{x}}$ but not $C_{\mathrm{p}}$, the capacitance $C_{\mathrm{p}}$ should be

$$
C_{\mathrm{p}} \geq 4 C_{\mathrm{x}} .
$$

When the high voltage $V_{s}$ is solely applied, the equivalent circuit is as shown in Fig. 2(b). For DC voltages, the branches of $C_{\mathrm{x}}$ and $C_{\mathrm{p}}$ can be considered as an open circuit. Therefore, the equivalent circuit is simplified as $R_{\mathrm{s}}$ in series with $R_{\mathrm{x}}$. Since $R_{\mathrm{x}}$ is much larger than $R_{\mathrm{s}}$, most of the applied DC voltage is undertaken by the sample. However, for AC voltages with a frequency of $f_{\mathrm{s}}$, the situation is more complicated since the branches of $C_{\mathrm{x}}$ and $C_{\mathrm{p}}$ can no longer be considered as an open circuit. The branch of $R_{\mathrm{X}}$ can be neglected as $R_{\mathrm{X}}$ is much larger than the impedance of other branches. The voltage 


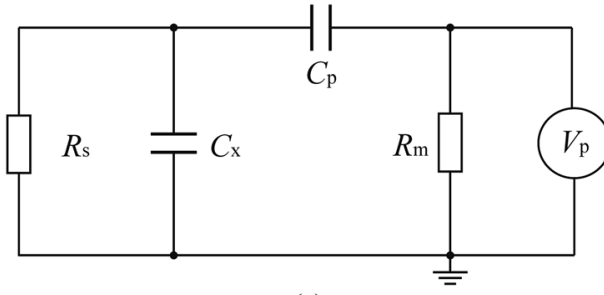

(a)

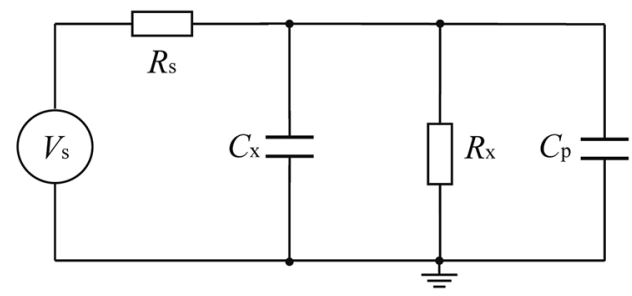

(b)
FIG. 2. The equivalent electric circuit at different circumstances. (a) The pulse voltage $V_{p}$ is solely applied. (b) The high voltage $V_{\mathrm{s}}$ is solely applied. undertaken by the sample is

$$
\overrightarrow{U_{\mathrm{x}}}=\vec{U}_{\mathrm{s}} \times \frac{1 / j 2 \pi f_{\mathrm{s}}\left(C_{\mathrm{p}}+C_{\mathrm{x}}\right)}{1 / j 2 \pi f_{\mathrm{s}}\left(C_{\mathrm{p}}+C_{\mathrm{x}}\right)+R_{\mathrm{s}}}=\vec{U}_{\mathrm{s}} \times \frac{1}{1+j 2 \pi f_{\mathrm{s}} R_{\mathrm{s}}\left(C_{\mathrm{p}}+C_{\mathrm{x}}\right)} .
$$

To guarantee that more than $80 \%$ of the AC voltage is undertaken by the sample, not the protection resistor $R_{s}$, the impedance of parallel $C_{\mathrm{x}}$ and $C_{\mathrm{p}}$ should be

$$
\begin{gathered}
\left|\vec{U}_{\mathrm{x}}\right|=\left|\vec{U}_{\mathrm{s}}\right| \times \frac{1}{\sqrt{1+\left[2 \pi f_{\mathrm{s}} R_{\mathrm{s}}\left(C_{\mathrm{p}}+C_{\mathrm{x}}\right)\right]^{2}}} \geq 0.8\left|\vec{U}_{\mathrm{s}}\right|, \\
C_{\mathrm{p}} \leq \frac{1}{2 \pi f_{\mathrm{s}}}\left(\frac{3}{4 R_{\mathrm{s}}}\right)-C_{\mathrm{x}} .
\end{gathered}
$$

From the above equation, $R_{\mathrm{s}}$ should be as small as possible in order to extend the suitable range of capacitance $C_{\mathrm{p}}$. From Eqs. (2) and (5), the suitable range of $C_{\mathrm{p}}$ is

$$
4 C_{\mathrm{x}} \leq C_{\mathrm{p}} \leq \frac{1}{2 \pi f_{\mathrm{s}}}\left(\frac{3}{4 R_{\mathrm{s}}}\right)-C_{\mathrm{x}} .
$$

Choosing appropriate values of $R_{\mathrm{s}}$ and $C_{\mathrm{p}}$ based on the abovementioned equations could make full usage of the voltage supplies. However, there still exist some differences between the voltage undertaken by the sample $U_{\mathrm{i}} \sin \left(2 \pi f_{\mathrm{i}} t+\varphi_{\mathrm{i}}\right)$, where $t$ is time, $U_{\mathrm{i}}$ is the amplitude, $f_{\mathrm{i}}$ is the frequency, and $\varphi_{\mathrm{i}}$ is the phase angle of the voltage undertaken by the sample, and the applied AC voltage $U_{\mathrm{si}} \sin \left(2 \pi f_{\mathrm{i}} t+\varphi_{\mathrm{si}}\right)$, where $U_{\mathrm{si}}$ is the amplitude and $\varphi_{\mathrm{si}}$ is the phase angle of the applied AC voltage, especially for high frequencies. The relationship is

$$
\begin{aligned}
U_{\mathrm{i}} \sin \left(2 \pi f_{\mathrm{i}} t+\varphi_{\mathrm{i}}\right) & =U_{\mathrm{si}} \sin \left(2 \pi f_{\mathrm{i}} t+\varphi_{\mathrm{si}}\right) \times \frac{1}{1+j 2 \pi f_{\mathrm{i}}\left(C_{\mathrm{p}}+C_{\mathrm{x}}\right) R_{\mathrm{s}}}, \\
U_{\mathrm{si}} & =U_{\mathrm{i}} \times \sqrt{1+\left[2 \pi f_{\mathrm{i}}\left(C_{\mathrm{p}}+C_{\mathrm{x}}\right) R_{\mathrm{s}}\right]^{2}}, \\
\varphi_{\mathrm{si}} & =\varphi_{\mathrm{i}}+\arctan \left[2 \pi f_{\mathrm{i}}\left(C_{\mathrm{p}}+C_{\mathrm{x}}\right) R_{\mathrm{s}}\right] .
\end{aligned}
$$

Therefore, for AC voltage $U_{i} \sin \left(2 \pi f_{i} t+\varphi\right)$ to be undertaken by the sample, the amplitude of the applied voltage should be $U_{i}\left\{1+\left[2 \pi f_{\mathrm{i}}\left(C_{\mathrm{p}}+C_{\mathrm{x}}\right)\right]^{2}\right\}$ and the phase angle should be $\left\{\varphi_{i}+\arctan \left[2 \pi f_{i}\left(C_{\mathrm{p}}+C_{\mathrm{x}}\right)\right]\right\}$. This is the calibration method when the applied voltage is a single-frequency sinusoid function.

For arbitrary waveform voltage $U_{\text {arb }}$, it can be decomposed to the sum of many sinusoid functions with different frequencies, amplitudes, and phase angles through Fast Fourier Transform (short as FFT),

$$
U_{\text {arb }}=\sum_{i=1}^{n} U_{\mathrm{i}} \sin \left(2 \pi f_{\mathrm{i}} t+\varphi_{\mathrm{i}}\right) .
$$

For each frequency component, Eqs. (8) and (9) are used for calibration. Then, the actual voltage waveform that needs to be applied can be obtained by summing up each component of the calibrated sinusoid function, which is as follows:

$$
U_{\text {app }}=\sum_{i=1}^{n} U_{\mathrm{i}}^{\prime} \sin \left(2 \pi f_{\mathrm{i}} t+\varphi_{\mathrm{i}}^{\prime}\right) \text {. }
$$

It is important to note that the influence of temperature and frequency on capacitance $C_{\mathrm{p}}$ and $C_{\mathrm{x}}$ should also be taken into consideration if its relative permittivity has an obvious change that cannot be neglected. Here, the blocking capacitor used in the system is not sensitive to either frequency or temperature.

\section{DESIGN OF THE NANOSECOND PULSE GENERATOR}

Space charge measurements under arbitrary waveform voltages pose new challenges to the PEA system. In order to capture the charge dynamics at different phase angles continuously and precisely, the pulse generator should be able to generate pulses with a higher amplitude, a higher repetition rate, a lower jitter, and a comparatively shorter width. Compared with most solid state switches, avalanche transistors are most suitable for this application due to its ultrafast switching speed, high hold-off voltage, low jitter, tiny size, and commercial availability. In this paper, a nanosecond pulse generator based on avalanche transistors is developed for the PEA system.

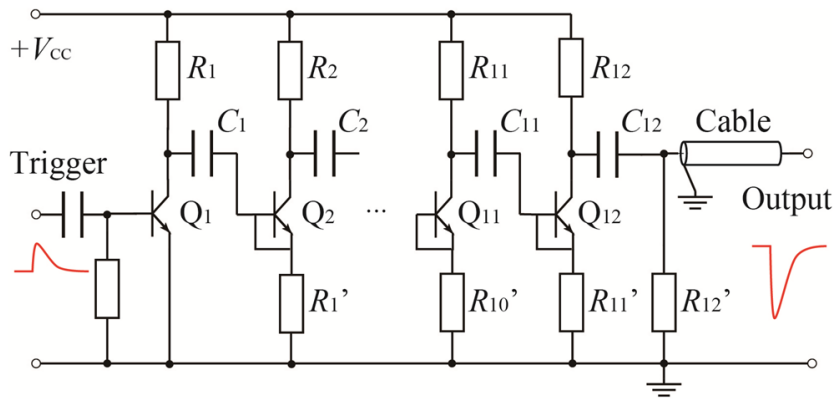

FIG. 3. The schematic diagram of the improved Marx generator based on avalanche transistors. 


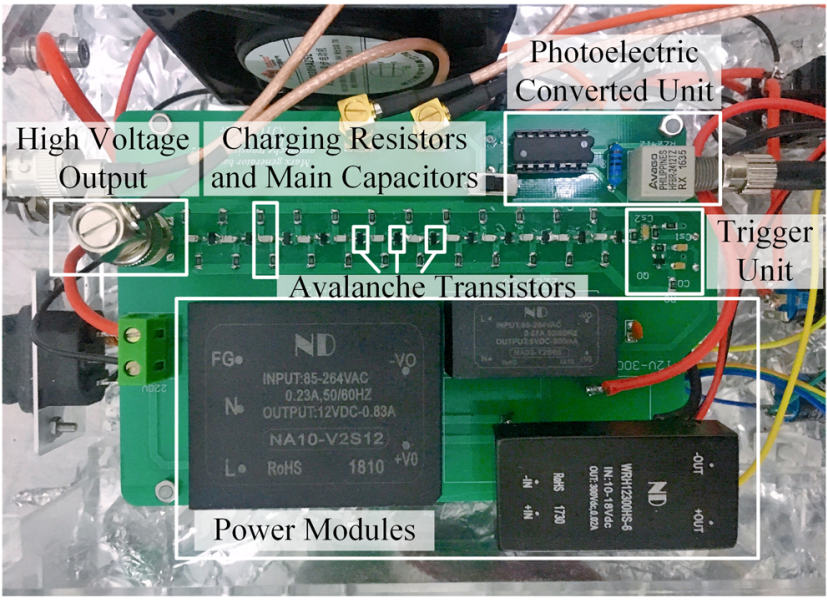

FIG. 4. The picture of the $1 \times 12$-stage Marx circuit based on avalanche transistors.

The schematic diagram of this nanosecond pulse generator is shown in Fig. 3, which consists of two main parts, the trigger unit and the Marx circuit. The trigger unit is also a small circuit based on the avalanche transistor and can produce a trigger pulse with a rise time of $5 \mathrm{~ns}$ and an amplitude of $60 \mathrm{~V}$ between the base and emitter of $\mathrm{Q}_{1}$ through capacitor $C_{0}$. In order to avoid the electromagnetic interference from the Marx circuit during the switching process of avalanche transistors, the $5 \mathrm{~V}$ transistor-transistor logic (TTL) signal from the microcontroller is transferred through a photoelectric transducer with the optical fiber. For the Marx circuit, it includes three key elements: the avalanche transistors $\mathrm{Q}_{1}-\mathrm{Q}_{12}$ (FMMT417, SOT-23 package), the main capacitors $C_{1}-C_{12}$ (Mica, in-line package, $100 \mathrm{pF}$ ), and the charging/isolating resistors $R_{1}-R_{12}$ and $R_{1}{ }^{\prime}-R_{12}{ }^{\prime}$ (1812 package, $10 \mathrm{k} \Omega$ ). Thus, the charging time constant, $\tau=\left(R_{1}+{R_{1}}^{\prime}\right) C_{1}$, is $\sim 2 \mu$ s and the maximal repetition rate, $f=(5 \tau)^{-1}$, is $\sim 100 \mathrm{kHz}$ when the speed of charging and cooling can be satisfied. The number of stages of the circuit is chosen as 12 considering the voltage superposition efficiency, and each stage is charged to $+300 \mathrm{~V}$. Except for $\mathrm{Q}_{1}$, the bases and emitters of all the other avalanche transistors are short-connected.
The operation principle of this generator can be summarized as follows: First, capacitors $C_{1}-C_{12}$ are charged to $300 \mathrm{~V}$ through the resistors $R_{1}-R_{12}$ and $R_{1}{ }^{\prime}-R_{12}{ }^{\prime}$, and are kept in a critical avalanche state. When a pulse needs to be generated, a 5 V TTL signal produced by the microcontroller and transmitted through the photoelectric transducer will be applied to the trigger unit. At this time, a fast trigger pulse is generated between the base and emitter of $Q_{1}$. After that, $\mathrm{Q}_{1}$ is switched on and its collector, i.e., the left end of $C_{1}$, is connected to the ground. When neglecting the residual voltage on the transistor, the potential at the right end of $C_{1}$ becomes $-300 \mathrm{~V}$ and the potential of $C_{2}$ remains $+300 \mathrm{~V}$ at the instant due to the existence of isolating resistors. Thus, $\mathrm{Q}_{2}$ bears almost $600 \mathrm{~V}$ and is switched on immediately under the overvoltage ramp. The avalanche transistors in the subsequent stages are switched on in a similar pattern. When all stages complete the switching process, a nanosecond high voltage pulse with negative polarity is generated on the load. Finally, after the charges in the capacitors are fully released, the capacitors will be charged again by $V_{\mathrm{CC}}$ and be ready for the generation of next pulse. It should be mentioned that the above analysis does not consider the residual voltage on the transistors, and it is assumed that the transistors in subsequent stages switch on almost simultaneously. Therefore, the actual overvoltage ramp on each transistor is smaller than two times of $V_{\mathrm{CC}}$ or it may destroy the transistors.

The generator is as shown in Fig. 4. The size of the printed circuit board (PCB) is only $130 \times 95 \mathrm{~mm}^{2}$. There are two control signal ports on the board. One is for the microcontroller built in the pulse generator that is usually used in occasions without phase correction. The other one is for the external microcontroller that can be synchronized with the arbitrary voltage waveform generated by the high voltage power supply. The output port of the generator is connected with the pulse input port of the electrode system through a $15 \mathrm{~m}$-long coaxial cable with an impedance of $50 \Omega$.

Figure 5 shows the output waveform of the pulse generator on a $50 \Omega$ resistive load. The amplitude is $\sim 700 \mathrm{~V}$ and the full width is $\sim 5$ ns. Here, we regard the standard deviation of the time difference between the rising edge of the trigger pulse and the rising edge of the output pulse as the time jitter. By testing and calculation, the time jitter is only $0.24 \mathrm{~ns}$, which indicates the great stability of this generator. Then, the generator is tested continuously for $2 \mathrm{~h}$ with a repetition rate of $10 \mathrm{kHz}$, and no failures are observed.

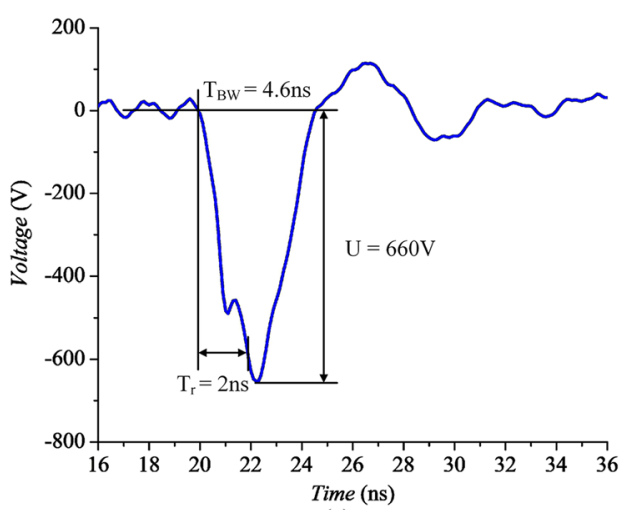

(a)

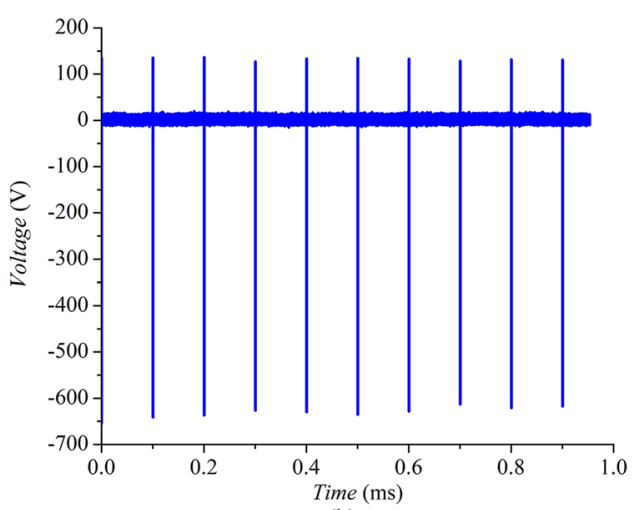

(b)
FIG. 5. The output pulses at the end of the cable. (a) The single pulse at $10 \mathrm{kHz}$ : the amplitude is $-660 \mathrm{~V}$ and the rise time is $2 \mathrm{~ns}$. (b) The pulses at $10 \mathrm{kHz}$. 


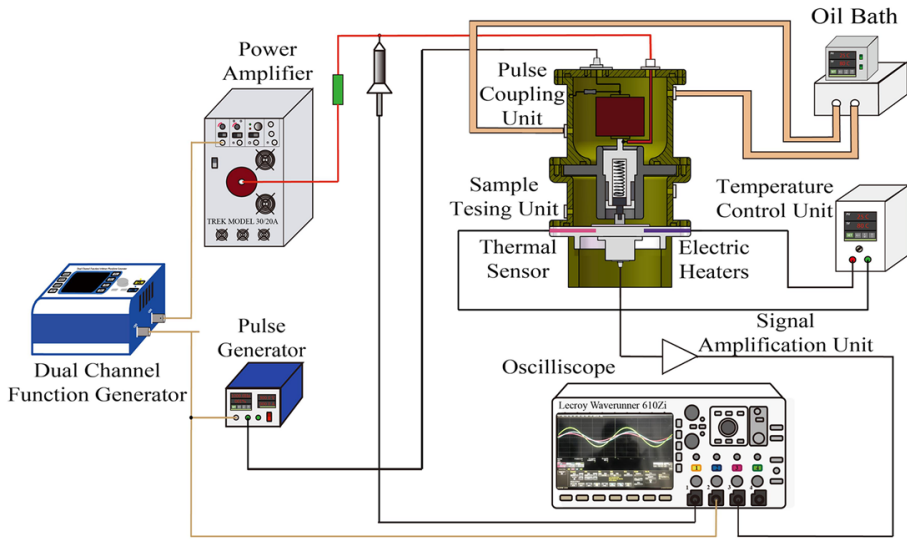

(a)

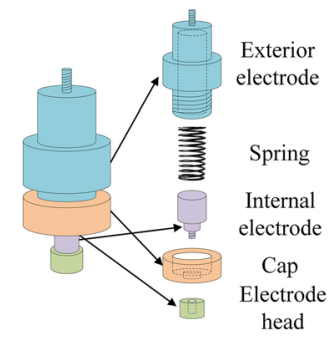

(b)
FIG. 6. The schematic diagram of the improved PEA measurement system. (a) General view. (b) The design of the flexible electrode unit.

\section{DESIGN OF THE IMPROVED PEA ELECTRODE SYSTEM}

The schematic diagram and the picture of the improved PEA measurement system are shown in Figs. 6(a) and 7, respectively. The pulse generator is externally triggered by channel 1 of the dual channel function generator. The applied voltage is generated by channel 2 of the dual channel function generator and the power amplifier. In order to achieve practical convenience, temperature consistency, and better repeatability of measurement results under high temperatures and arbitrary waveform voltages, the electrode system has been redesigned. The improved electrode system can be divided into four units: pulse coupling unit, sample testing unit, temperature control unit, and signal transmission-amplification unit.

The pulse coupling unit, installed in the upper chamber, consists of the matching resistor $R_{\mathrm{m}}$ and the blocking capacitor $C_{\mathrm{p}}$. Usually, for the convenience of insulation and fixing, an epoxy resin is used to cast the $R C$ circuit in the upper chamber. In that way, neither the $R C$ unit nor the high voltage electrode can be replaced. As analyzed in Sec. II, when applying voltages with different waveforms on different samples, the blocking capacitor $C_{\mathrm{p}}$ needs to be changed

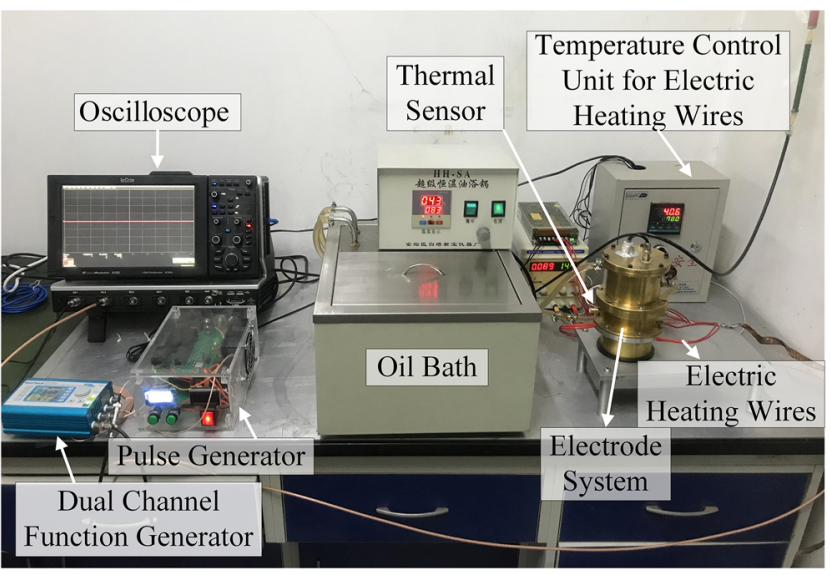

FIG. 7. The picture of the improved PEA measurement system. from time to time. Instead of pouring, the upper chamber in this paper is insulated by transformer oil. In this way, its internal components can be easily replaced. This provides great convenience for PEA tests under arbitrary waveform voltages.

In traditional design, the blocking capacitor, the matching resistor, the input line of pulses, and the upper electrode were casted into a metal cylindrical chamber by the epoxy resin. The upper electrode system was usually placed at a concentric place with the sample. Then, the whole electrode system was fixed by the mounting screws. On the one hand, keeping the upper electrode system horizontal is very important. Since if one side of the upper electrode is slightly higher than the other side, the close contact between the sample and the upper electrode cannot be guaranteed. On the other hand, the force of external screws is a rigid connection to some extent. It is difficult to control the force on the screws to be exactly the same each time. For hard samples, like the epoxy resin, this would result in a different tightness of connection between the sample and the electrodes. The tightness of contact has a great influence on the transmission of acoustic signals, which further compromises the accuracy and repeatability of testing results. For soft samples, like polyethylene, different forces bring about different deformations of the sample, which further results in a different field strength. To overcome this shortcoming, an improved flexible electrode unit, which is installed in the middle chamber, is designed and developed, as shown in Fig. 6 . The high voltage electrode is split into five parts: the external electrode, the spring, the internal electrode, the cap, and the electrode head. Specially, the external electrode is with a hollow structure. The spring and the internal electrode are both mounted inside the outer electrode and fixed by the cap. The internal electrode can compress the spring and move along the axis. Besides, the internal electrode and the electrode head are connected by screw threads, so the electrode heads with different diameters and materials can be easily replaced. This design can be used to study the influence of different electrode materials and sizes on the charge injection characteristics. Meanwhile, when the thickness of the sample varies between hundreds of micrometers and several millimeters, the compression of the spring barely changes. Therefore, the force applied on the sample is basically constant. This ensures the consistency of the measurement conditions and the stability of the experimental results. 
Space charge measurement at high temperatures requires strict consistency of temperature near the sample. Any small temperature difference between the upper and lower surface will lead to a large space-resolved testing error of the space charge. The traditional heating methods, which include wrapping a heating band around or putting the whole device into a high temperature oven, cause problems of low heating efficiency and difficulty in changing the samples. Here, we adopt the hybrid heating method of oil bath and electric heating wires. Six holes are drilled evenly on the side of the grounded aluminum electrode plate. Five of them are used to install the electric heating wires and the other one is used to install the temperature sensor. The external temperature control circuit monitors the temperature sensor and guarantees the constant temperature of the grounded electrode through a negative feedback control. At the same time, high temperature silicone oil is being circulated continuously in the upper chamber. Measurement results on the temperatures of the upper surface of the sample and the grounded electrode show that there is no temperature difference, which could guarantee the temperature consistency around the sample. It only takes around $10 \mathrm{~min}$ to rise from room temperature to $80^{\circ} \mathrm{C}$. On the other hand, the heating area is concentrated near the sample, which can avoid interference and influence on temperature sensitive elements, such as the amplifier.

The most sensitive part of the whole PEA system is the signal transmission-amplification unit. The PVDF/TrFE film (Piezotech $\mathrm{FC}^{\mathrm{TM}} 25$ ) with a thickness of $20 \mu \mathrm{m}$ is adopted as the piezoelectric sensor due to its higher Curie temperature and better heat tolerance, which can function well at $100^{\circ} \mathrm{C}$. The upper surface of the film is in close contact with the lower surface of the aluminum electrode. The piezoelectric transducer converts acoustic signals into voltage signals, which is amplified by a high-bandwidth, low-noise amplifier. It is important to note that the state of the interface between the film and the electrode has a great influence on the test signal. Thus, the installation of the piezoelectric sensor should be very careful.

To summarize, the improved PEA electrode system in this paper has three main innovations:

(1) The flexible electrode unit. This ensures that the contact between the electrodes and the sample is almost the same in each experiment and guarantees the consistency of measurement conditions.
(2) The hybrid heating method of oil bath and electric heating wires. While ensuring no temperature difference exists near the sample, it has a higher efficiency of heating. In the meantime, it will cause minimum influence on other temperaturesensitive devices, like the amplifier, since the heating area is very concentrated.

(3) The replaceable coupling capacitor and high voltage electrode head. This provides convenience not only for the accurate measurement of charge dynamics under arbitrary waveform voltages but also for the long-term maintenance.

\section{EXPERIMENTAL MEASUREMENTS}

\section{A. Measurements at different temperatures}

In order to test the performance of the measurement system at different temperatures, especially high temperatures, space charge measurements were conducted on both low density polyethylene and epoxy resin samples, the thicknesses of which are around $210 \mu \mathrm{m}$ and $250 \mu \mathrm{m}$, respectively. The measurement results at different temperatures are shown in Fig. 8.

As it can be seen, the shapes of the waveforms seem alike, which all include a negative peak followed by a positive overshoot and a positive peak followed by a negative overshoot. The negative peak represents the capacitive charges near the lower electrode, while the positive peak corresponds to the capacitive charges near the higher electrode. The overshoot following the peak is closely related to the system's response. In order to eliminate the influence of overshoots and obtain an accurate charge distribution inside the sample, the measured waveforms need to be calibrated by using a reference of the system's response. Since attenuation and dispersion might influence the transmission of acoustic signals through the sample, the peak and the following overshoot near the lower electrode is used as the reference. The similarity of waveforms proves that the system is functioning properly at different temperatures.

There are some differences in the amplitude of waveforms and their positions on the time axis. To be more specific, with the increasing temperature, the waveform gradually shifts to the right direction, the amplitude decreases, and the waveform becomes wider. This phenomenon is similar to previous experimental observations, which is caused by the decreased acoustic velocity with the increasing temperature. ${ }^{28}$ Since the reference waveform used in

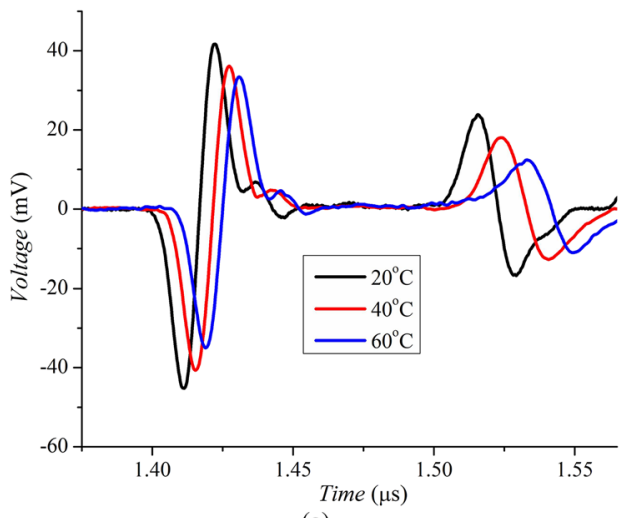

(a)

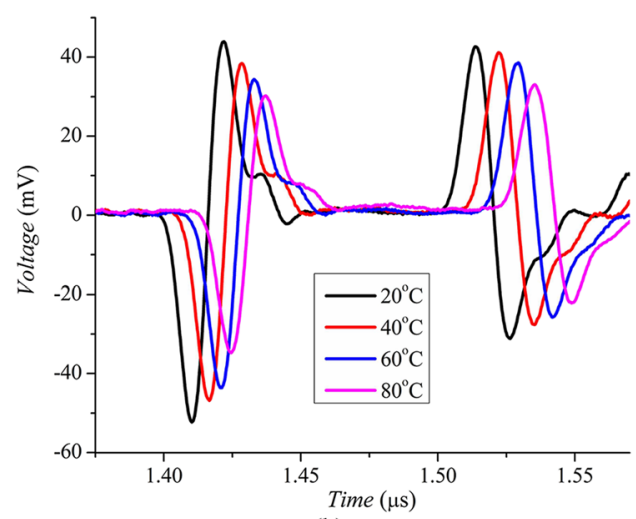

(b)
FIG. 8. The measurement waveforms of space charge at different temperatures. (a) Low density polyethylene. (b) Epoxy resin. 


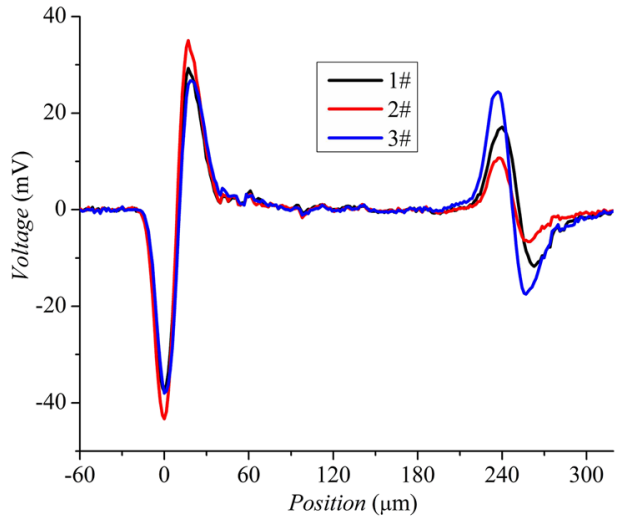

(a)

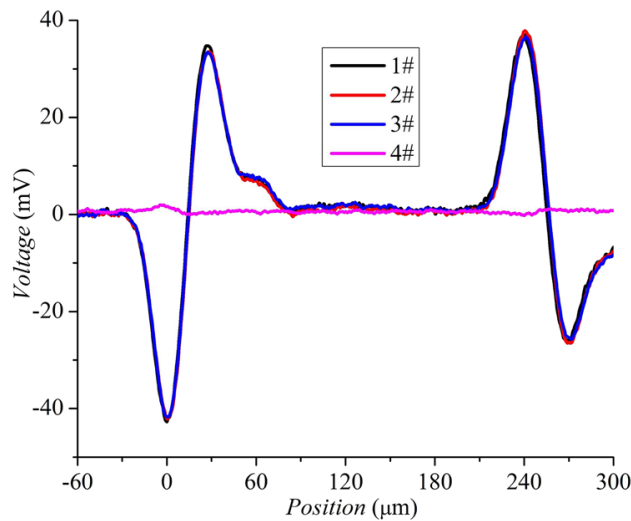

(b)
FIG. 9. The comparison on the consistency of measurement results (a) using the old electrode system and (b) using the improved flexible electrode. space charge calibration is measured at the same temperature, the influence of temperature can be eliminated.

By comparing Fig. 8(a) with Fig. 8(b), the shape difference between the first negative peak and the second positive peak is larger in polyethylene than that in the epoxy resin, which means that the attenuation and dispersion of acoustic signals transmitted through polyethylene is larger than that through the epoxy resin. Furthermore, for polyethylene, the temperature dependence of attenuation and dispersion of acoustic signals is also larger than that for the epoxy resin.

\section{B. Validation of the flexible electrode unit}

The close and consistent contact between the sample and the electrodes is very important for the transmission of acoustic signals. In order to verify that the improved flexible electrode with a spring could guarantee a better contact between the sample and the electrodes, the measurements were conducted on the epoxy resin sample at the same field strength of $10 \mathrm{kV} / \mathrm{mm}$ for three times using the old and the improved upper electrode. Specifically, the old upper electrode system is not horizontal when it is placed on the sample.

The results are shown in Fig. 9. As shown in Fig. 9(a), there is a huge difference in the signals at the upper electrode. This is because when using the old system, there is one side of the upper electrode that is higher than the other side. In this case, the poor contact severely compromises the consistency of measurement results. Furthermore, the way of applying fixing forces through external screws provides a rigid connection. Since the applied forces are difficult to control precisely, the contact between the sample and the electrodes is hardly consistent each time. In contrast, the improved electrode unit ensures the close and consistent contact, and the results are highly consistent in the three measurements, as shown in Fig. 9(b).

In addition, the fourth waveform in Fig. 9(b) is when the pulse voltage is solely applied without any DC stresses. It could be seen that the influence of the pulse voltage itself is negligible, which proves that the amplitude of the pulses is not too high.

\section{Measurements under arbitrary waveform voltages}

From previous experiments, the improved PEA system can measure space charge distributions accurately in a wide temperature range under DC voltages. As for measurements under AC voltages, first, the blocking capacitor $C_{\mathrm{p}}$ should be carefully chosen based on Eq. (6), which requires the capacitance of the sample $C_{\mathrm{x}}$. The sample used in the following measurements was an epoxy resin with a thickness of $250 \mu \mathrm{m}$. At $20^{\circ} \mathrm{C}$, the relative permittivity of the epoxy resin between $100 \mathrm{~Hz}$ and $500 \mathrm{~Hz}$ is around 3.4. The area of the

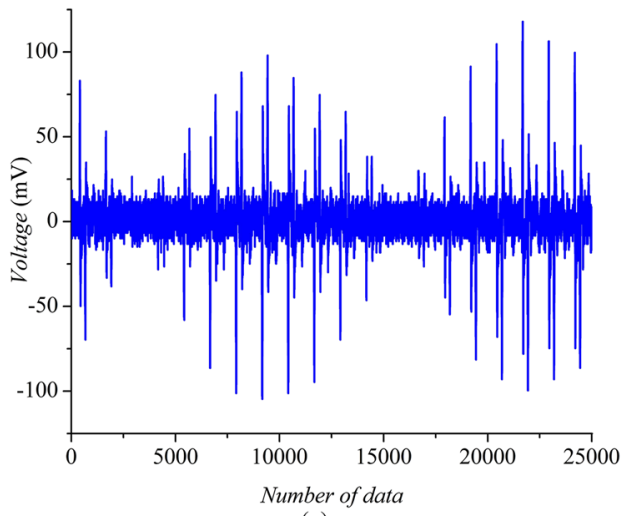

(a)

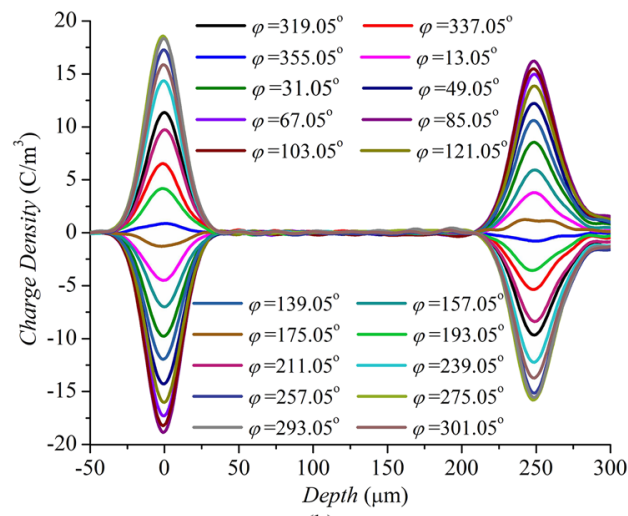

(b)
FIG. 10. The measurement results under $500 \mathrm{~Hz}$ AC voltage. (a) The acquired data in one cycle. (b) The charge distributions at different phase angles. 


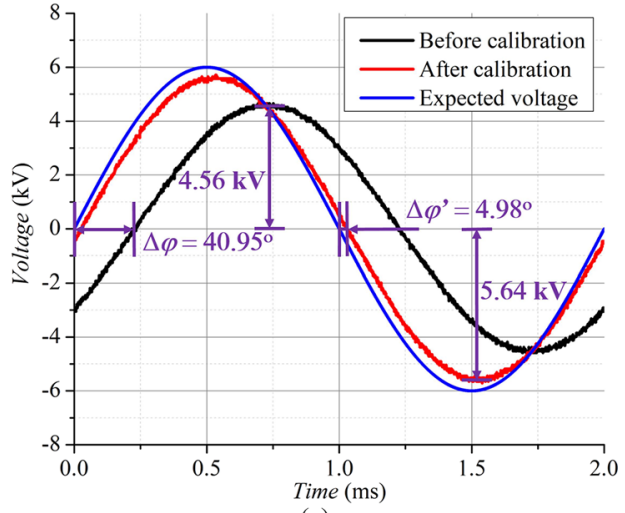

(a)

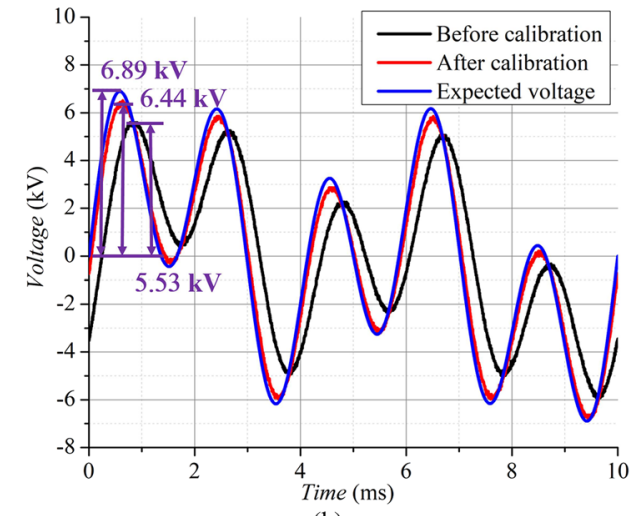

(b)
FIG. 11. The variation of $V_{1}, V_{2}$, and $\rho_{1}$ with time (a) before calibration and (b) after calibration. upper electrode is $2.0 \mathrm{~cm}^{2}$. The capacitance of the sample $C_{\mathrm{x}}$ is $24.2 \mathrm{pF}$. Based on Eq. (6), a capacitor with a nominal value of $200 \mathrm{pF}$ is selected as the blocking capacitor. Through measurements using a bridge, the actual capacitance of $C_{\mathrm{p}}$ is $204.3 \mathrm{pF}$. Second, the oscilloscope is switched to "sequence" mode to realize continuous data acquisition at different phase angles. Due to the low signal-to-noise ratio, charge profiles should be averaged with the ones measured at the same phase angle in the following cycles for at least 20 times to suppress the interferences of noises. In this paper, charge profiles at 20 different phase angles were measured and 100 cycles of data were acquired and recorded each time.

When the applied voltage is a $500 \mathrm{~Hz}$ AC voltage with a peak value of $6 \mathrm{kV}$, the repetitive frequency of the pulse generator is set as $10 \mathrm{kHz}$. The acquired data in one cycle consist of 20 segments of data, as shown in Fig. 10(a). After averaging and calibration, the acquired charge distributions are shown in Fig. 10(b). The results proved that the improved PEA system could realize a precise measurement of charge profiles at different phase angles when a high frequency AC voltage is applied. Furthermore, the first segment of space charge data is not measured at phase angle $0^{\circ}$. There is a difference of phase angle between the applied voltage, which is the output voltage of the power amplifier and function generator, and the voltage undertaken by the sample, which is in accordance with our theoretical analysis in Sec. II.

In order to measure the voltage undertaken by the sample more accurately, a high voltage probe was connected to the high voltage electrode, as shown in Fig. 6 . The expected voltage undertaken by the sample is $6000 \times \sin (2 \pi \times 500 \times t)$. Before voltage calibration, the applied voltage is set as $6000 \times \sin (2 \pi \times 500 \times t)$. After the voltage calibration based on Eqs. (8) and (9), the applied voltage is set as $7380 \times \sin \left(2 \pi \times 500 \times t+35.57^{\circ}\right)$. The voltages undertaken by the sample before and after calibration are measured, respectively, as shown in Fig. 11(a). The amplitudes and phase angles of the voltages obtained through fitting are shown in Table I. Before calibration, the deviation of phase angle from the expected voltage is $40.95^{\circ}$. The amplitude is about $24.05 \%$ smaller. In comparison, after calibration, the difference in phase angle decreased from $40.95^{\circ}$ to $4.98^{\circ}$, and the deviation of amplitude decreased from $24.05 \%$ to $6.05 \%$, which proved the effectiveness of the theoretical analysis and the calibration method.

For voltages with arbitrary waveforms, they can be decomposed to the sum of many sinusoidal voltage components. A voltage with three frequency components: $1500 \times \sin (2 \pi \times 100 \times t)$, $3000 \times \sin (2 \pi \times 200 \times t)$ and $4500 \times \sin (2 \pi \times 500 \times t)$ is taken as an example to be the expected voltage. The voltage undertaken by the sample before and after calibration is shown in Fig. 11(b), and the parameters obtained through fitting are shown in Table I. The voltage waveform undertaken by the sample is distorted before calibration. The maximum positive voltage is $5.53 \mathrm{kV}$, while that of the expected voltage is $6.89 \mathrm{kV}$. For each frequency component, the deviation from the expected voltage is different. The deviation becomes larger at higher frequencies, which is reasonable according

TABLE I. Parameters of the voltage components before and after calibration

\begin{tabular}{|c|c|c|c|c|}
\hline \multicolumn{2}{|r|}{ Items } & \multirow{2}{*}{$\frac{\text { Frequency } f(\mathrm{~Hz})}{500}$} & \multirow{2}{*}{$\frac{\text { Amplitude }|\mathrm{A}|(\mathrm{V})}{4557}$} & \multirow{2}{*}{$\begin{array}{c}\text { Phase Angle } \varphi(\mathrm{deg}) \\
-40.95\end{array}$} \\
\hline $500 \mathrm{~Hz}$ AC voltage & Before calibration & & & \\
\hline 500 HZ AC voilage & After calibration & 500 & 5637 & -4.98 \\
\hline \multirow{6}{*}{$\begin{array}{l}\text { An arbitrary voltage with } \\
\text { three AC components }\end{array}$} & Component 1: before calibration & 100 & 1465 & -8.74 \\
\hline & Component 1: after calibration & 100 & 1487 & -1.09 \\
\hline & Component 2: before calibration & 200 & 2814 & -18.09 \\
\hline & Component 2: after calibration & 200 & 2942 & -3.69 \\
\hline & Component 3: before calibration & 500 & 3396 & -42.24 \\
\hline & Component 3: after calibration & 500 & 4187 & -6.68 \\
\hline
\end{tabular}


to Eqs. (8) and (9). After calibration, the deviation from the expected voltage significantly decreased, which also proved the feasibility of the proposed method of voltage calibration for arbitrary waveforms. However, there are still some minor deviations caused by the stray capacitances, for instance, the silicone oil inside the sample testing unit and the pulse coupling unit, and loop inductances of the measurement system, which were not taken into consideration in the theoretical analysis. Since the stray capacitances and loop inductances are really hard to calculate or measure accurately and the deviation is comparatively small, its influences can be neglected.

\section{CONCLUSIONS}

In this paper, an improved PEA system used for space charge measurements under high temperatures and arbitrary voltage waveforms was developed and a voltage calibration method was proposed to realize accurate voltage application under arbitrary voltage waveforms, especially for the applied voltages containing high frequency components. The sampling rate, the resolution, and the accuracy of the measurement system are enhanced by developing the novel pulse supply that can generate pulses with an amplitude of $700 \mathrm{~V}$, a width of $4.6 \mathrm{~ns}$, and a repetitive frequency of $10 \mathrm{kHz}$. The improvement on the electrode system mainly consists of three parts: the more efficient way of heating through a combination of oil bath and electric heaters, the flexible electrode unit with a spring that guarantees the close contact between the sample and the electrodes, and the replaceable blocking capacitor that provides great practical convenience. Through experimental validation, the improved PEA system presents a more stable and consistent performance under various temperatures. Despite some minor deviations, the proposed method of voltage calibration could effectively solve the problem of large deviation from the expected voltage.

\section{ACKNOWLEDGMENTS}

The authors would like to thank the staff working in Tony Davies High Voltage Laboratory for their kind support. This work was supported by the Fundamental Research Funds for the Central Universities (Grant No. xzy012019029) and the Shaanxi Key Research and Development Program of China (Grant No. 2018ZDCXL-GY-07-03).

\section{REFERENCES}

${ }^{1}$ N. B. Negra, J. Todorovic, and T. Ackermann, Electr. Power Syst. Res. 76, 916-927 (2006).

${ }^{2} \mathrm{~K}$. Meah and S. Ula, in IEEE Power Engineering Society General Meeting (IEEE, 2007), pp. 1-5.
${ }^{3}$ B. V. Eeckhout, D. V. Hertem, M. Reza, K. Srivastava, and R. Belmans, Eur Trans. Electr. Power 20, 661-671 (2010).

${ }^{4} \mathrm{~J}$. A. C. Forrest and B. Allard, in IEEE Power Engineering Society General Meeting (IEEE, 2003), p. 1271003.

${ }^{5}$ Q. Wang, J. Liao, H. Tian, P. Liu, and Z. Peng, IEEE Trans. Dielectr. Electr. Insul. 24(5), 3254-3264 (2017).

${ }^{6}$ S. Koch, H. D. Gersem, T. Weiland, J. Hoppe, and P. Heinzig, Electr. Eng. 90, 331-336 (2008).

${ }^{7}$ W. Choo, G. Chen, and S. G. Swingler, IEEE Trans. Dielectr. Electr. Insul. 18(2), 596-606 (2014).

${ }^{8}$ J. Zhao, G. Chen, and L. Zhong, IEEE Trans. Dielectr. Electr. Insul. 21(4), 1757-1763 (2014).

${ }^{9}$ B. Huang, M. Hao, J. Hao, J. Fu, Q. Wang, and G. Chen, IEEE Trans. Dielectr. Electr. Insul. 23(2), 881-891 (2016).

${ }^{10}$ T. Maeno, T. Futami, H. Kushibe, T. Takada, and C. M. Cooke, IEEE Trans. Dielectr. Electr. Insul. 23(3), 433-439 (1988).

${ }^{11}$ Y. Li, M. Yasuda, and T. Takada, IEEE Trans. Dielectr. Electr. Insul. 1(2), 188-195 (1994).

${ }^{12}$ Y. Zhang, J. Lewiner, C. Alquie, and N. Hampton, IEEE Trans. Dielectr. Electr. Insul. 3(6), 778-783 (1996).

${ }^{13}$ J. Hao, G. Chen, R. Liao, L. Yang, and C. Tang, IEEE Trans. Dielectr. Electr. Insul. 19(4), 1456-1464 (2012).

${ }^{14}$ H. Mu, D. Wang, S. Wang, J. Tian, Y. Li, Y. Wei, and G. Zhang, High Vol. Eng. 39(8), 1865-1872 (2013).

${ }^{15} \mathrm{H}$. Kitajima, Y. Tanaka, and T. Takada, in VIIth International Conference on Dielectric Materials Measurements and Applications (IEEE, 1996), pp. 8-11.

${ }^{16}$ J. C. Fothergill, L. A. Dissado, J. Alison, and A. See, in VIIIth International Conference on Dielectric Materials, Measurements and Applications (IEEE, 2000), pp. 352-356.

${ }^{17}$ X. Chen, X. Wang, K. Wu, Z. R. Peng, Y. H. Cheng, and D. M. Tu, IEEE Trans. Dielectr. Electr. Insul. 17(6), 1796-1805 (2010).

${ }^{18} \mathrm{H}$. Wang, K. Wu, Q. Zhu, and X. Wang, IEEE Trans. Dielectr. Electr. Insul. 22(2), 1213-1219 (2015).

${ }^{19}$ Z. Lv, J. Cao, X. Wang, H. Wang, K. Wu, and L. A. Dissado, IEEE Trans. Dielectr. Electr. Insul. 22(6), 3186-3196 (2015).

${ }^{20}$ X. Wang, N. Yoshimura, Y. Tanaka, K. Murata, and T. Takada, J. Phys. D: Appl. Phys. 31(16), 2057-2064 (1998).

${ }^{21} \mathrm{Z}$. Xu, J. Zhao, and G. Chen, in 2010 International Conference on Solid Dielectrics (IEEE, 2010), pp. 1-4.

${ }^{22} \mathrm{~J}$. Wu and Y. Yin, IEEE Trans. Dielectr. Electr. Insul. 24(4), 2579-2588 (2017).

${ }^{23}$ Y. Li, J. Kawai, Y. Ebinuma, and Y. Fujiwara, IEEE Trans. Dielectr. Electr. Insul. 4(1), 52-57 (1997).

${ }^{24}$ G. C. Montanari, G. Mazzanti, E. Boni, and G. D. Robertis, in International Conference on Electrical Insulation and Dielectric Phenomena (IEEE, 2000), pp. 113-116.

${ }^{25}$ M. Fukuma, T. Takao, and M. Fujii, in International Conference on Electrical Insulation and Dielectric Phenomena (IEEE, 2011), pp. 157-160.

${ }^{26}$ S. Bamji, M. A. Dakka, and A. Bulinski, IEEE Trans. Dielectr. Electr. Insul. 14(1), 77-82 (2007).

${ }^{27}$ S. Bamji, A. Fujita, A. Bulinski, and M. A. Dakka, IEEE Trans. Dielectr. Electr. Insul. 14(5), 1110-1112 (2007).

${ }^{28}$ X. Chen, X. Wang, K. Wu, Z. Peng, and Y. Cheng, Acta Phys. Sin. 59(10), 7327-7332 (2010). 